\title{
ÜBER EINE ASYMPTOTISCHE DARSTELLUNG DER LÖSUNG EINES SYSTEMS VON DIFFERENTIALGLEICHUNGEN, WELCHE VON ZWEI PARAMETERN ABHÄNGEN, UND EINE ANWENDUNG AUF SPEZIELLE DIFFERENTIALGLEICHUNGEN ZWEITER ORDNUNG
}

\author{
KEN-ICHI TAKAHASHI
}

(Received March 22, 1960)

In dieser Abhandlung möchte ich eine von Parametern abhängende asymptotische Darstellung der Lösung des Systems von homogenen linearen Differentialgleichungen, welche von zwei Parametern abhängen, in einer einfacher Weise erledigen, und diese Theorie auf spezielle Differentialgleichungen zweiter Ordnung anwenden.

Schon hat Herr Prof. M. Hukuhara eine asymptotische Darstellung der Lösung eines Systems von homogenen linearen Differentialgleichungen erörtert, welche von einem Parameter abhängen." Aber kann man im allgemeinen die asymptotische Lösung eines Systems von Differentialgleichungen, welche von zwei Parametern abhängen, nicht erledigen, wenn die charakteristische Gleichung ${ }^{2)}$ eine mehrfache Wurzel besitzt. Um also die asymptotische Lösung in allen Fällen, wie in [1], zu erfinden, müssen wir den Begriff der bisherigen asymptotischen Entwickelung erweitern.

Es ist bezweckt in dieser Abhandlung erstens in $\S 1$ die bisherige asymptotische Entwickelung zu erweitern; zweitens in $\$ 2$ den Existenzsatz zu erklären; und schliesslich in $\S \S 3,4$ und 5 diese Theorie auf der Besselschen Differentialgleichung und der konfluenten hypergeometrischen Differentialgleichung zu anwenden.

Unser Theorie lässt sich sicher allgemeinen auf Differentialgleichungen mit einer regulären singulären Stelle $x=0$ und mit einer irregulären singulären Stelle $x=\infty$ anwenden, aber wir können diese Theorie auf eine Differentialgleichung mit dem Wendepunkt ("Turning point" auf englisch) nicht anwenden.

Neuerdings hat Herr M. Iwano eine neue Theorie aufgestellt, welche sich auf eine Differentialgleichung mit dem Wendepunkt anwenden lässt. ${ }^{3)}$ In dieser Abhandlung erkläre ich die Definition der asymptotischen Entwickelung und den

1) Vgl. [1].

2) Vgl. [4], §2,4०.

3) Vgl. [2]. 
Existenzsatz, welche von Herrn M. Iwano verbessert worden sind.

\section{§ 1. Definition der asymptotischen Entwickelung.}

1. Definition des Halbstrahls $L_{r}$ und des Zeichens $K_{r}$. Wir setzen für $n, n^{\prime} ; n_{0}, n_{0}^{\prime}$ voraus, dass sie sämtlich natürliche Zahlen sind, dass $n$ und $n^{\prime}$ usw. zueinander prim sind und dass die folgende Ungleichung gilt:

$$
N_{0}=n n_{0}^{\prime}-n^{\prime} n_{0}>0 \text {. }
$$

Es seien :

$$
\begin{array}{ll}
L: & n_{0}^{\prime} x+n_{0} y=0,(x \geqq 0), \\
L_{r}: & n^{\prime} x+n y+r=0,\left(x \leqq r n_{0} / N_{0}\right) .
\end{array}
$$

Man bestimme $x_{r}$ und $y_{r}$ durch

$$
\begin{aligned}
x_{r}= & \text { Max }\left[\text { Ganze Zahlen } x, \text { wofür } x \leqq r n_{0} / N_{0}\right. \text { gilt, und } \\
& \left(n^{\prime} x+r\right) / n \text { eine ganze Zahl wird], } \\
y_{r}= & -\left(n^{\prime} x_{r}+r\right) / n .
\end{aligned}
$$

Dann definiert man für solche $x_{r}$ und $y_{r}$ das Zeichen $K_{r}\left(n, n^{\prime} ; n_{0}, n_{0}^{\prime} ; \lambda, \mu\right)$ durch $\mathrm{K}_{r}\left(n, n^{\prime} ; n_{0}, n_{0}^{\prime} ; \lambda, \mu\right)=\lambda^{y_{r}} \mu^{x_{r}}{ }^{4)}$

2. Definition der asymptotischen Entwickelung. Von jetzt an benutzt man die folgenden Zeichen :

$$
\begin{array}{ll}
D: & |x|<r, \\
\Delta_{\lambda}: & \alpha<\arg \lambda<\alpha^{\prime}, R<|\lambda|<\infty, \\
\Delta_{\mu}: & \beta<\arg \mu<\beta^{\prime}, R^{\prime}<|\mu|<\infty, \\
\Delta_{\nu}: & \gamma<\arg \boldsymbol{\nu}<\boldsymbol{\gamma}^{\prime} .0<|\boldsymbol{\nu}|<R^{\prime \prime} .
\end{array}
$$

Es gelte die folgende Ungleichung zwischen den Ordnungen von $\lambda$ und $\mu$ :

$$
U_{0}: \quad K|\lambda|^{\sigma}<|\mu|<\frac{1}{K^{\prime}}|\lambda|^{\sigma_{0}},
$$

wo $\sigma$ und $\sigma_{0}$, $\left(0<\sigma<\sigma_{0}\right)$ rationale Zahlen, und $K$ und $K^{\prime}$ hinreichend grosse positive Zahlen sind. Man bestimmt zueinander prime, natürliche Zahlen $n, n^{\prime}$; $n_{0}, n_{0}^{\prime}$ derart, dass $n^{\prime} / n=\sigma, n_{0}^{\prime} / n_{0}=\sigma_{0}$.

Es sei $f(x, \lambda, \mu)$ für $\left(D, \Delta_{\lambda}, \Delta_{\mu}, U_{0}\right)$ regulär, und die folgende Ungleichung gelte für alle natürlichen Zahlen $N$ grösser als eine natürliche Zahl $N_{1}$ :

$$
\left|f(x, \lambda, \mu)-\sum_{r=0}^{N-1} K_{r} f_{r}\left(x, \lambda^{n^{\prime}} \mu^{-n}\right)\right| \leqq K_{N}\left(|\lambda|^{-\frac{n_{0}^{\prime}}{N_{0}}}|\mu|^{\frac{n_{0}}{N_{0}}}\right)_{, N}^{N}(\lambda \rightarrow \infty, \mu \rightarrow \infty),
$$

4) Der Einfachheit halber schreibt man an Stelle von $K_{r}\left(n, n^{\prime} ; n_{0}, n_{0}^{\prime} ; \lambda, u\right)$ bloss $K^{r}$. 
worin $f_{r}(x, \nu)$ für $\left(D, \Delta_{\nu}\right)$ regulär sind, ${ }^{5)}$ und sie sich für $\nu \rightarrow 0$ folgendermassen asymptotisch entwickeln lassen:

$$
f_{r}(x, \nu) \simeq \sum_{s=0}^{\infty} f_{r s}(x) \nu^{s},(r=0, \ldots \ldots, N-1),
$$

wo $f_{r s}(x)$ in $D$ regulär sind. Dann sagen wir dass $f(x, \lambda, \mu)$ sich für $\lambda \rightarrow \infty$, $\mu \rightarrow \infty$ in die Gestalt:

$$
f(x, \lambda, \mu) \simeq \sum_{r=0}^{\infty} K_{r} f_{r}\left(x, \lambda^{n^{\prime}} \mu^{-n}\right)
$$

asymptotisch entwickeln lässt.

\section{§ 2. Existenzsatz.}

3. Existenzsatz. Wir denken uns das System

$$
d z_{j} / d x=\lambda^{m} \mu^{m^{\prime}}\left[f_{j}(x, \lambda, \mu) z_{j}+\sum_{k=1}^{n} b_{j k}(x, \lambda, \mu) z_{k}\right],(j=1, \ldots \ldots, n),
$$

wo $m$ und $m^{\prime}$ ganze Zahlen sind. Man stellt die folgenden Voraussetzungen:

(i) Es ist $m_{0}=m n+m^{\prime} n^{\prime}>0, m n_{0}+m^{\prime} n_{0}^{\prime} \geqq 0{ }^{6)}$ wo $n, n^{\prime} ; n_{0}, n_{0}^{\prime}$ zueinander prime, natürliche Zahlen von der Art sind, dass die Ungleichung $N_{0}=n n_{0}^{\prime}-n^{\prime} n_{0}>0$ gilt.

(ii) Die Funktionen $f_{j}(x, \lambda, \mu)$ sind für $\left(D, \Delta_{\lambda}, \Delta_{\mu}, U_{0}\right)$ regulär, und

$$
\left.f_{j}(x, \lambda, \mu)=\sum_{r=0}^{N+m_{0}} K_{r} f_{j}\left(x, \lambda^{n^{\prime}} \mu^{-n}\right)\right)^{7)}
$$

wo $f_{j}(x, \nu)$ für $\left(D, \Delta_{\nu}\right)$ regulär sind, und sie sich für $\nu \rightarrow 0$ folgendermassen asymptotisch entwickeln lassen :

$$
f_{j}(x, \nu) \simeq \sum_{s=0}^{\infty} f_{j s}(x) \nu^{s},
$$

wo $\underset{r s}{f_{j}}(x)$ in $D$ regulär sind. Die Zahl $N$ ist eine später zu bestimmende passende natürliche $Z_{\text {ahl }}{ }^{8)}$

(iii) Die Funktionen $b_{j k}(x, \lambda, \mu)$ sind für $\left(D, \Delta_{\lambda}, \Delta_{\mu}, U_{0}\right)$ regulär, und sie lassen sich für $\lambda \rightarrow \infty, \mu \rightarrow \infty$ folgendermassen asymptotisch entwickeln :

5) Wir müssen das Bereich $\Delta_{\nu}$ derart annehmen, dass $\lambda^{n \prime} \mu^{-n} \in \Delta_{\nu}$ ist.

6) Der Beweis des im Falle $m_{0}=0$ gültigen Existenzsatzes wurde von Herrn M. Iwano [2] gegeben.

7) Wenn wir an Stelle von $U_{0}$ und $K_{r}$ bzw. $U_{1}$ und $K_{r, 1}$ annehmen, dann ist der Existenzsatz ebenfalls richtig. Vgl. [2]. Bald definieren wir die Zeichen $U_{1}$ und $K_{r, 1}$.

8) Vgl. die Voraussetzung (v) des Artikels 3. 


$$
b_{j k}(x, \lambda, \mu) \simeq \sum_{r=N+m_{0}+1}^{\infty} K_{r} b_{j k}\left(x, \lambda^{n^{\prime}} \mu^{-n}\right) .
$$

(iv) Wir nehmen für eine Kurve $\Gamma$ wie an:

(a) Die Kurve $\Gamma$ ist in $D$ enthalten und geschlossen.

(b) Die Kurve $\Gamma$ besitzt eine Anzahl von Ecken.

(c) Die Kurve $\Gamma$ lässt sich durch eine Funktion $x(t)$ darstellen, die als Periode die Länge von $\Gamma$ besitzt, die eine von der Länge $t$ des Bogens von $\Gamma$ abhängende, stetige Funktion ist, und die bis auf an den Ecken stetig differentierbar ist.

Das Bereich $D^{*}$ sei das Innere von $\Gamma$. Es gilt die folgende Ungleichung:

$$
\begin{aligned}
& \Re\left[x^{\prime}(t)\left(\lambda^{m} \mu^{m \prime} f_{j}(x, \lambda, \mu)+g_{j}(x)\right)\right]\left\{\begin{array}{l}
\leqq-\varepsilon,\left(x \in \widehat{x_{j} \xi_{j}}, \Delta_{\lambda}, \Delta_{\mu}, U_{0}\right), \\
\geqq \quad \varepsilon,\left(x \in \widehat{\xi_{j} x_{j}}, \Delta_{\lambda}, \Delta_{\mu}, U_{0}\right),
\end{array}\right. \\
& (j=1, \ldots \ldots, n),
\end{aligned}
$$

wo $\varepsilon>0$ ist, und $g_{j}(x)$ in $D$ regulär sind.

(v) Die Zahl $N+m_{0}+1$ ist ein Multiplum von $N_{0}$, und $N$ ist eine natürliche $Z$ ahl. Die natürlichen Zahlen $n$ und $n^{\prime}$ sind zueinander prim, und sie werden durch

$$
\frac{n_{1}^{\prime}}{n_{1}}=\frac{\frac{n_{0}^{\prime}}{N_{0}}\left(N+m_{0}+1\right)-m}{\frac{n_{0}}{N_{0}}\left(N+m_{0}+1\right)+m^{\prime}}\left(\equiv \sigma_{1}\right)
$$

bestimmt. Das Zeichen $K_{r, 1}$ lässt sich durch $K_{r, 1}=K_{r}\left(n, n^{\prime} ; n_{1}, n_{1}^{\prime} ; \lambda, \mu\right)$ definieren."

(vi) Das System (3.1) besitzt eine formale Lösung von der Gestalt :

$$
z_{j} \approx \sum_{r=0}^{\infty} K_{r, 1} p_{j}\left(x, \lambda^{n^{\prime}} \mu^{-n}\right),(j=1, \ldots \ldots, n),
$$

wo $p_{r}(x, \nu)$ für $\left(D, \Delta_{\nu}\right)$ regulär sind, und sie sich für $\nu \rightarrow 0$ folgendermassen asymptotisch entwickeln lassen :

$$
p_{j}(x, \nu) \simeq \sum_{s=0}^{\infty} p_{j}(x) \nu^{s}
$$

worin $\underset{r s}{p_{j}}(x)$ in $D$ regulär sind. Überdies gilt für alle $r\left(\geqq N^{*}\right)$ die Beziehung :

$$
\lambda^{m} \mu^{m \prime} K_{\dot{r}, 1} p_{j}(x, \nu)=K_{r-m_{0,1}} \underset{r}{p_{j}^{*}}(x, \nu),
$$

9) Man kann $\sigma_{1}$ derart annehmen, dass $\sigma_{0}-\sigma_{1}<\varepsilon,(\varepsilon,(>0)$ sei sehr klein. $)$ ist, wenn man $N$ hinreichend gross $z u$ sein annimmt. 
wo $p_{r}^{*}(x, \nu)$ dieselben Eigenschaft wie $p_{r}(x, \nu)$ besitzen, wenn man eine hinreichend grosse natürliche Zahl $N^{*}$ aufnimmt. ${ }^{10)}$

Unter der Voraussetzungen (i), (ii), (iii), (iv), (v) und (vi) besitzt das System (3. 1) dann eine eindeutige Lösung, welche für $\left(D, \Delta_{\lambda}, \Delta_{\mu}, U_{1}\right)$ regulär ist, und sich für $\lambda \rightarrow \infty, \mu \rightarrow \infty$ folgendermassen asymptotisch entwickeln lässt:

$$
z_{j}(x, \lambda, \mu) \simeq \sum_{r=0}^{\infty} K_{r, 1} p_{r}\left(x, \lambda^{n^{\prime}} \mu^{-n}\right),(j=1, \ldots \ldots, n),
$$

wo

$$
U_{1}: \quad K|\lambda|^{\sigma}<|\mu|<\frac{1}{K^{\prime}}|\lambda|^{\sigma_{1}}
$$

ist. $^{11)}$

4. Formale Lösung. Wir denken uns ein System von hornogenen linearen Differentialgleichungen : ${ }^{12}$

$$
d y_{j} / d x=\lambda^{m} \mu^{m^{\prime}} \sum_{k=1}^{n} a_{j k}(x, \lambda, \mu) y_{k},(j=1, \ldots \ldots, n),
$$

die sich mit Benutzung einer Matrize folgenderweise umschreiben lässt :

$$
d Y / d x=\lambda^{m} \mu^{m^{\prime}} A(x, \lambda, \mu) Y,
$$

wo $m$ und $m^{\prime}$ ganze Zahlen sind. Wir stellen die folgenden Voraussetzungen :

(i) Es gilt die. Voraussetzung (i) des Artikels 3.

(ii) Es ist $A(x, \lambda, \mu)$ für $\left(D, \Delta_{\lambda}, \Delta_{\mu}, U_{0}\right)$ regulär, und $A(x, \lambda, \mu)$ lässt sich für $\lambda \rightarrow \infty, \mu \rightarrow \infty$ folgendermassen asymptotisch entwickeln :

$$
A(x, \lambda, \mu) \simeq \sum_{r=0}^{\infty} K_{r} A\left(x, \lambda^{n^{\prime}} \mu^{-n}\right),
$$

wo $A_{r}(x, \nu)$ sich für $\nu \rightarrow 0$ folgendermassen asymptotisch entwickeln lassen :

$$
\underset{r}{A}(x, \nu) \simeq \sum_{s=0}^{\infty} A(x) \nu^{s} .
$$

(iii) Es ist $A_{0}(x, \nu)$ eine diagonale Matrize mit

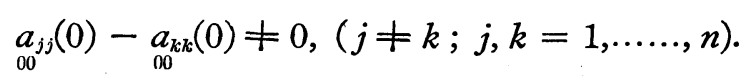

10) Vgl. [2].

11) Den Beweis dieses Existenzsatzes hat Herr M. Iwano in [1] gezeigt.

12) Das System (4.1) mit den Voraussetzungen (i), (ii) und (iii) wollen wir die Normalform nennen. 
Unter den Voraussetzungen (i), (ii) und (iii) reduzieren wir (4.1) zur Differentialgleichung von der Gestalt (3.1) des Artikels 3.

Es sei die Zahl $N+m_{0}+1$ ein Multiplum von $N_{0}$, und wir nehme eine minimale natürliche $Z$ ahl $N$ von der $A r t$ an, dass für eine gegebene positive rationale Zahl $\sigma_{1}\left(\leqq \sigma_{0}\right)$ die folgende Ungleichung gilt:

$$
\sigma_{1} \leqq \frac{\frac{n_{0}^{\prime}}{N_{0}}\left(N+m_{0}+1\right)-m}{\frac{n_{0}}{N_{0}}\left(N+m_{0}+1\right)+m^{\prime}} .
$$

Wendet man die Transformation

$$
Y=P(x, \lambda, \mu) Z
$$

mit

$$
P(x, \lambda, \mu)=\sum_{r=0}^{N+m_{0}} K_{r} P\left(x, \lambda^{n^{\prime}} \mu^{-n}\right)
$$

auf (4.1) an, wo man nach

$$
\begin{gathered}
{\left[\lambda^{m} \mu^{m^{\prime}} \sum_{s=0}^{r} \sum_{m=0}^{r-s} K_{s} K_{m} K_{r-s-m} \underset{s}{Q}(x, \nu) \underset{m}{A}(x, \nu) \underset{r-s-m}{P}(x, \nu)\right.} \\
\left.-\sum_{s=0}^{r-m_{0}-1} K_{s} K_{r-m_{0}-s} \underset{s}{Q}(x, \nu) \underset{r-m_{0}-s}{P^{\prime}}(x, \nu)\right]_{j k}=0, \\
\left(j \neq k ; j, k=1, \ldots \ldots, n ; r=1, \ldots \ldots, N+m_{0}\right), \\
\underset{r}{P}(x, \nu)]_{j j}=0,\left(j=1, \ldots \ldots, n ; r=1, \ldots \ldots, N+m_{0}\right)
\end{gathered}
$$

$P_{r}(x, \nu),\left(r=1, \ldots \ldots, N+m_{0}\right)$ nacheinander bestimmt, ${ }^{13)}$ so geht $(4.1)$ ins Folgende über :

$$
d z_{j} / d x=\lambda^{m} \mu^{m^{\prime}}\left[f_{j}(x, \lambda, \mu) z_{j}+\sum_{k=1}^{n} b_{j k}(x, \lambda, \mu) z_{k}\right],(j=1, \ldots \ldots, n) .
$$

Hierbei besitzen $f_{j}(x, \lambda, \mu)$ und $b_{j k}(x, \lambda, \mu)$ die folgenden Eigenschaften :

(a) Die Funktionen $f_{j}(x, \lambda, \mu)$ sind für $\left(D, \Delta_{\lambda}, \Delta_{\mu}, U_{0}\right)$ regulär und

$$
f_{j}(x, \lambda, \mu)=\sum_{r=0}^{N+m_{0}} K_{r} f_{j}\left(x, \lambda^{n^{\prime}} \mu^{-n}\right),
$$

wo $f_{r}(x, \nu)$ für $\left(D, \Delta_{\nu}\right)$ regulär sind, und sie sich für $\nu \rightarrow 0$ folgendermassen asymptotisch entwickeln lassen :

13) Betreffs des Falls $m_{0}=0$ ist die Bestimmung von $\underset{r}{\underset{r}{P}(x, \nu)}$ in [2] ausführlich erklärt. 


$$
f_{j}(x, \nu) \simeq \sum_{s=0}^{\infty} f_{j s}(x) \nu^{s}
$$

wo $\underset{r s}{f_{j}}(x)$ in $D$ regulär sind.

(b) Die Funktionen $b_{j k}(x, \lambda, \mu)$ sind für $\left(D, \Delta_{\lambda}, \Delta_{\mu}, U_{0}\right)$ regulär, und lassen sich für $\lambda \rightarrow \infty, \mu \rightarrow \infty$ folgendermassen asymptotisch entwickeln :

$$
b_{j k}(x, \lambda, \mu) \simeq \sum_{r=N+m_{0}+1}^{\infty} K_{r} b_{r k}\left(x, \lambda^{n^{\prime}} \mu^{-n}\right) .
$$

Daheı genügt das System (4.5) den Voraussetzungen (i), (ii) und (iii) des Artikels 3, und ferner nach der Voraussetzung (iii) dieses Artikels gilt das in (iv) des Artikels 3 vorausgesetzte, wenn man den Bereich $D, \Delta_{\lambda}, \Delta_{\mu}$ und die Funktionen $g_{j}(x)$ passend auswählt.

Wendet man ferner die Transformation

$$
Z=\bar{P}(x, \lambda, \mu) \bar{Z}
$$

mit

$$
\bar{P}(x, \lambda, \mu) \approx E+\sum_{r=N+m_{0+1}}^{\infty} K_{r} \bar{P}\left(x, \lambda^{n^{\prime}} \mu^{-n}\right)
$$

auf (4.5) an, wo man nach

$$
\begin{aligned}
& {\left[\lambda^{m} \mu^{m^{\prime}} \sum_{s=0}^{r} \sum_{m=0}^{r-s} K_{s} K_{m} K_{r-s-m} \bar{s}(x, \nu) \underset{m}{B}(x, \nu) \underset{r-s-m}{\bar{P}}(x, \nu)\right.}
\end{aligned}
$$

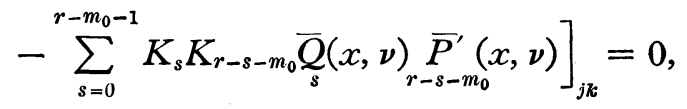

$$
\begin{aligned}
& \left(j \neq k ; j, k=1, \ldots \ldots, n ; r=N+m_{0}+1, \ldots \ldots\right), \\
& {[\vec{P}(x, \nu)]_{j j}=0,\left(j=1, \ldots \ldots, n ; r=N+m_{0}+1, \ldots \ldots\right)}
\end{aligned}
$$

$\bar{P}(x, \nu),\left(r=N+m_{0}+1, \ldots \ldots\right)$ nacheinander bestimmt, so geht (4. 5) ins Folgende über :

$$
d \overline{z_{j}} / d x=\lambda^{m} \mu^{m^{\prime}}\left[f_{j}(x, \lambda, \mu)+\bar{b}_{j}(x, \lambda, \mu)\right] \overline{z_{j}},(j=1, \ldots \ldots, n),
$$

wobei

(a') die Funktionen $\bar{b}_{j}(x, \lambda, \mu)$ sich folgendermassen formell entwickeln lassen :

$$
\overline{b_{j}}(x, \lambda, \mu) \approx \sum_{r=N+m_{0}+1}^{\infty} K_{r} \bar{b}_{j}\left(x, \lambda^{n^{\prime}} \mu^{-n}\right),
$$

$\left(\mathrm{b}^{\prime}\right)$ die Funktionen $\bar{b}_{r}(x, \nu)$ für $\left(D, \Delta_{\nu}\right)$ regulär sind, und sie sich folgender- 
massen asymptotisch entwickeln lassen :

$$
\bar{b}_{r}(x, \nu) \simeq \sum_{s=0}^{\infty} \overline{r_{j}}(x) \nu^{s},
$$

wo $\underset{r_{s}}{\overline{b_{j}}}(x)$ in $D$ regulär sind.

Wendet man ferner die Transformation

$$
\bar{Z}=\overline{\bar{P}}(x, \lambda, \mu) \overline{\bar{Z}}
$$

mit

$$
\overline{\bar{P}}(x, \lambda, \mu) \approx E+\sum_{r=N+1}^{\infty} \overline{\bar{P}}(x, \lambda, \mu)
$$

auf (4.8) an, wo man nach

$$
\begin{aligned}
\left.\underset{r}{\left[\overline{\bar{P}}^{\prime}\right.}(x, \lambda, \mu)\right]_{j j}= & {\left[\lambda^{m} \mu^{m^{\prime}} K_{r+1} \bar{B}\left(x, \lambda^{n^{\prime}} \mu^{-n}\right)-\sum_{s=N+1}^{r-N-1} \overline{\bar{Q}}(x, \lambda, \mu) \underset{r-s}{\bar{P}^{\prime}}(x, \lambda, \mu)\right]_{j j}, } \\
& (j=1, \ldots \ldots, n ; r=N+1, \ldots \ldots), \\
\underset{r}{[\overline{\bar{P}}}(x, \lambda, \mu)]_{j k}= & 0,(j \neq k ; j, k=1, \ldots \ldots, n ; r=N+1, \ldots \ldots)
\end{aligned}
$$

$\overline{\bar{P}}(x, \lambda, \mu),(r=N+1, \ldots \ldots)$ nacheinander bestimmt, so geht (4.5) ins Folgende über :

$$
d \overline{\overline{z_{j}}} / d x=\lambda^{m} \mu^{m \prime} f_{j}(x, \lambda, \mu) \overline{\overline{z_{j}}},(j=1, \ldots \ldots, n) .
$$

Aus (4. 10) folgt :

$$
\underset{r}{\bar{P}}(x, \lambda, \mu)=K_{r, 1} \underset{r}{\overline{\bar{P}}}\left(x, \lambda^{n^{\prime}} \mu^{-n}\right),
$$

worin $K_{r, 1}$ das in Artikel 3 definierte Zeichen ist, und $\underset{r}{\stackrel{\bar{P}}{(}}(x, \nu)$ für $\left(D, \Delta_{\nu}\right)$ regulär sind, und sich $\nu \rightarrow 0$ folgendermassen asymptotisch entwickeln lassen :

$$
\underset{r}{\overline{\bar{P}}}(x, \nu) \simeq \sum_{s=0}^{\infty} \overline{\bar{P}}(x) \nu^{s},
$$

wo $\underset{r s}{\overline{\bar{P}}}(x)$ in $D$ regulär sind; daher besitzt das System (4.5) die folgende formale Lösung:

(4. 12) $z_{j} \approx C_{k} \exp \left[F_{k}(x, \lambda, \mu)\right]\left[\delta_{j k}+\sum_{r=N+1}^{\infty} K_{r, 1} p_{r k}^{*}\left(x, \lambda^{n^{\prime}} \mu^{-n}\right)\right],(j=1, \ldots \ldots, n)$.

$\left(\mathrm{a}^{\prime \prime}\right)$ Es ist $F_{k}(x, \lambda, \mu)=\lambda^{m} \mu^{m^{\prime}} \int f_{k}(x, \lambda, \mu) d x$ 
$\left(b^{\prime \prime}\right)$ Die Funktionen $p_{r}^{*}(x, \nu)$ sind für $\left(D, \Delta_{\nu}\right)$ regulär, und lassen sich für $\nu \rightarrow 0$ folgendermassen asymptotisch entwickeln:

$$
p_{r k}^{*}(x, \nu) \simeq \sum_{s=0}^{\infty} p_{k i k}^{*}(x) \nu^{s}
$$

wo $\underset{r s}{p_{j k}^{*}}(x)$ in $D$ regulär sind.

( $\left.{ }^{\prime \prime}\right)$ Wenn man eine hinreichend grosse natürliche Zahl $N^{*}\left(\geqq m_{0}\right)$ aufnimmt, dann für alle $r\left(\geqq N^{*}\right)$ gilt

$$
\lambda^{m} \mu^{m \prime} K_{r, 1} p_{r}^{* k}(x, \nu)=K_{r-m_{0}, 1}^{*} p_{r}^{* *}(x, \nu),
$$

wo $\underset{r}{p_{j k}^{* *}}(x, \nu)$ dieselben Eigenschaft wie $\underset{r}{p_{j k}^{*}}(x, \nu)$ besitzen.

Durch die Transformation

$$
z_{j}=\hat{z}_{j} \exp \left[F_{k}(x, \lambda, \mu)\right],(j=1, \ldots \ldots, n)
$$

wird (4.5) $\mathrm{zu}$

$$
d \hat{Z} / d x=\lambda^{m} \mu^{m^{\prime}}\left[\left(F(x, \lambda, \mu)-f_{k}(x, \lambda, \mu) E\right)+B(x, \lambda, \mu)\right] \hat{Z},
$$

die eine für $\left(D, \Delta_{\lambda}, \Delta_{\mu}, U_{1}\right)$ reguläre eindeutige Lösung $\hat{z}_{j}(x, \lambda, \mu)$ besitzt, die sich folgendermassen asymptotisch entwickeln lässt :

$$
\hat{z}_{j}(x, \lambda, \mu) \simeq \delta_{j k}+\sum_{r=N+1}^{\infty} K_{r, 1} p_{r k}^{*}\left(x, \lambda^{n^{\prime}} \mu^{-n}\right),(j=1, \ldots . ., n) ;
$$

daher erhält man nach (4.3) eine asymptotische Lösung von (4.1). ${ }^{14}$

\section{§ 3. Die Besselsche Differentialgleichung.}

5. Reduktion zur Normalform. Wir betrachten die Besselsche Differentialgleichung :

$$
\hat{x}^{2} \frac{d^{2} \hat{y}}{d \hat{x}^{2}}+\hat{x} \frac{d \hat{y}}{d \hat{x}}+\left(\hat{x}^{2}-\lambda^{2}\right) \hat{y}=0
$$

mit einer irregulären singulären Stelle $x=\infty$, wo $\lambda$ ein Parameter ist. Es ist bezweckt für hinreichend grosse absolute Beträge von $\hat{x}$ und $\lambda$ eine asymptotische Darstellung der Lösung des Systems (5.1) nachzusuchen. Der Einfachheit halber betrachten wir den Fall, dass die Ungleichung

$$
M|\lambda|<|\hat{x}|<\frac{1}{M^{\prime}}|\lambda|^{\sigma_{0}}
$$

14) Die ausführliche Berechnung der formalen Lösung ist entweder in [2] oder in [5] gezeigt. 
gilt, wo die Grössen $M$ und $M^{\prime}$ hinreichend gross sind, und die $\sigma_{0}(>1)$ eine rationale $Z$ ahl ist.

Führt man die Transformation $\hat{x}=\mu x$ in (5.1) ein, wobei wir an Stelle von $\hat{x}$ als unabhängige Veränderliche die Variable $x$ von der Art aufnehmen, dass

$$
D: \quad|x-a|<r,(r<|a|)
$$

ist, wo die Grösse $a$ von Null verschieden ist, so geht (5.1) ins Folgende über :

$$
x^{2} \frac{d^{2} \hat{y}}{d x^{2}}+x \frac{d \hat{y}}{d x}+\left(x^{2} \mu^{2}-\lambda^{2}\right) \hat{y}=0,
$$

welche weiter durch die Transformation $\hat{y}=\hat{y}_{2}, d \hat{y} / d x=\mu \hat{y}_{1} \mathrm{zu}$

$$
d \hat{Y} / d x=\mu \hat{A}(x, \lambda, \mu) \hat{Y}
$$

wird, wo

$$
\hat{A}(x, \lambda, \mu)=\left(\begin{array}{cc}
-\frac{1}{\mu x} & -1+\frac{\lambda^{2}}{\mu^{2} x^{2}} \\
1 & 1
\end{array}\right)
$$

ist. Man kann die Ungleichung

$$
U_{0}: \quad K|\lambda|<|\mu|<\frac{1}{K^{\prime}}|\lambda|^{\sigma_{0}}
$$

ohne Schwierigkeit aus (5.2) herleiten. Daraus folgt:

$$
n=1, n^{\prime}=1, \frac{n_{0}^{\prime}}{n_{0}}=\sigma_{0} .
$$

Wir haben schon gezeigt, dass die Normalform eine asymptotische Lösung besitzt. Um das System (5.4) zur Normalform reduzieren zu lassen, wenden wir die Transformation

$$
\hat{Y}=P_{1}(x, \lambda, \mu) Y
$$

mit

$$
\begin{aligned}
P_{1}(x, \lambda, \mu) & =\left(\begin{array}{cc}
\rho\left(x, \lambda \mu^{-1}\right) & -\rho\left(x, \lambda \mu^{-1}\right) \\
1 & 1
\end{array}\right), \\
\rho(x, \nu) & =\sqrt{\frac{\nu^{2}-x^{2}}{x^{2}}}
\end{aligned}
$$

auf (5.4) an, so geht (5.4) ins Folgende über:

$$
d Y / d x=\mu A(x, \lambda, \mu) Y,
$$


wo

$$
\begin{gathered}
A(x, \lambda, \mu)=\underset{0}{A}\left(x, \lambda \mu^{-1}\right)+\lambda^{-1} A_{1}\left(x, \lambda \mu^{-1}\right), \\
\underset{0}{A}(x, \nu)=\left(\begin{array}{cc}
\rho(x, \nu) & 0 \\
0 & -\rho(x, \nu)
\end{array}\right), \underset{1}{A}(x, \nu)=\left(\begin{array}{cc}
\frac{\nu}{2 x \rho^{2}(x, \nu)} & -\frac{\nu}{2 x \rho^{2}(x, \nu)} \\
-\frac{\nu}{2 x\left(\rho^{2} x, \nu\right)} & \frac{\nu}{2 x \rho^{2}(x, \nu)}
\end{array}\right)
\end{gathered}
$$

ist ; daher ist das System (5.5) sicher eine Normalform.

6. Asymptotische Lösung. Wir betrachten nochmals das System (5.5) mit (5.6). Durch das ähnliche Verfahren wie bei Artt. 3 und 4 können wir die asymptotische Lösung des Systems (5.3) erledigen.

Durch die Transformation

$$
Y=P(x, \lambda, \mu) Z
$$

mit

$$
P(x, \lambda, \mu)=\sum_{r=0}^{N+1} K_{r} P\left(x, \lambda \mu^{-1}\right)
$$

geht (5.5) in

(6. 2) $\quad d z_{j} / d x=\mu\left[f_{j}(x, \lambda, \mu) z_{j}+\sum_{k=1}^{2} b_{j k}(x, \lambda, \mu) z_{k}\right],(j=1,2)$

über, welche genau wie bei $\S 2$ für $k=1,2$ zwei Paare unabhängiger formaler Lösungen besitzt :

$$
z_{j} \approx C_{k} \exp \left[F_{k}(x, \lambda, \mu)\right]\left[\delta_{j k}+\sum_{r=N+1}^{\infty} K_{r, 1} p_{r}^{*}\left(x, \lambda, \mu^{-1}\right)\right],(j=1,2),
$$

welche uns durch $\hat{Y}=P_{1}(x, \lambda, \mu) P(x, \lambda, \mu) Z$ die formale Lösung von (5. 4) liefert.

Von jetzt an behandeln wir der Einfachheit halber ausschliesslich den Fall $\sigma_{0}=3, \sigma_{1}=3 / 2$. In diesem Falle gilt offenbar die Beziehung :

$$
\frac{\frac{n_{0}^{\prime}}{N_{0}}\left(N+m_{0}+1\right)-m}{\frac{n_{0}}{N_{0}}\left(N+m_{0}+1\right)+m^{\prime}}=\sigma_{1},
$$

wenn wir $N=0$ annehmen. Wie eine kurze Berechnung zeigt, erhält man nach (4.3), (4.6) und (4.9) insbesondere 


$$
\begin{aligned}
& p_{12}(x, \nu)=\frac{\nu}{4 x \rho^{3}(x, \nu)}, p_{11}(x, \nu)=-p_{12}(x, \nu), p_{11}(x, \nu)=p_{22}(x, \nu)=0, \\
& \bar{p}_{12}(x, \nu)=\frac{\nu p_{12}^{\prime}(x, \nu)}{2 \rho(x, \nu)}, \bar{p}_{21}(x, \nu)=-\frac{\nu p_{12}^{\prime}(x, \nu)}{2 \rho(x, \nu)} \\
& \bar{p}_{21}(x, \nu)=\bar{p}_{22}(x, \nu)=0 \\
& \overline{\bar{p}}_{11}(x, \lambda, \mu)=-K_{1,1} \frac{\lambda^{2} \mu^{-2}(3+\cot \gamma)}{24 i \tan \gamma},\left(x=\frac{\lambda \mu^{-1}}{\cos \gamma}\right), \\
& \overline{\bar{p}}_{22}(x, \lambda, \mu)=-\overline{\bar{p}}_{11}(x, \lambda, \mu), \overline{\bar{p}}_{12}(x, \lambda, \mu)=\overline{\bar{p}}_{12}(x, \lambda, \mu)=0 .
\end{aligned}
$$

Ohne Schwierigkeit sehen wir dass die folgenden Beziehungen gelten :

$$
\begin{aligned}
& \exp \left[F_{1}(x, \lambda, \mu)\right]=\frac{1}{\sqrt{\lambda \mu^{-1} \tan \gamma}} \exp [i \lambda(\tan \gamma-\gamma)], \\
& \exp \left[F_{2}(x, \lambda, \mu)\right]=\frac{1}{\sqrt{\lambda \mu^{-1} \tan \gamma}} \exp [-i \lambda(\tan \gamma-\gamma)] .
\end{aligned}
$$

Das System (5.4) besitzt also eine eindeutige Lösung $\hat{y}(x, \lambda, \mu)$, die für ( $D$, $\left.\Delta_{\lambda}, \Delta_{\mu}, U_{1}\right)$ regulär ist, und sich folgendermassen asymptotisch entwickeln lässt:

$$
\begin{aligned}
\hat{y}(x, \lambda, \mu) \simeq & \sqrt{\frac{2}{\pi \lambda^{2} \mu^{-1} \tan \gamma}} \exp \left[i \lambda(\tan \gamma-\gamma)-\frac{\pi i}{4}\right] \\
& \times\left[1+\sum_{r=1}^{\infty} K_{r, 1} p_{r}\left(x, \lambda, \mu^{-1}\right)\right], \quad\left(x=\frac{\lambda \mu^{-1}}{\cos \gamma}\right),
\end{aligned}
$$

welches im speziellen Falle mit dem von Herrn Prof. T. Inui gezeigten Resultate übereinstimmt, ${ }^{15)}$ wo

$$
\begin{array}{r}
C_{1}=\sqrt{\frac{2}{\pi \lambda}} \exp \left(-\frac{\pi i}{4}\right), \\
U_{1}: \quad K|\lambda|<|\mu|<\frac{1}{K^{\prime}}|\lambda|^{\frac{3}{2}},
\end{array}
$$

und insbesondere

$$
p_{1}(x, \nu)=\frac{\nu^{5}\left(3+5 \cot ^{2} \gamma\right)}{24 i \tan \gamma}
$$

ist. Wie klein die Zahl $\sigma$ und wie gross die Zahl $\sigma^{\prime}$ auch sein mag, können wir für die Ungleichung

15) Vgl. [4]. 


$$
U_{2}: \quad K|\lambda|^{\sigma}<|\mu|<\frac{1}{K^{\prime}}|\lambda|^{\sigma^{\prime}}
$$

an Stelle von $U_{1}$ durch ein analoges Verfahren beweisen, dass das System (5.4) eine asymptotische Lösung besitzt.

\section{§4. Die konfluente hypergeometrische Differentialgleichung.}

7. Reduktion zur Normalform. Wir betrachten die konfluente hypergeometrische Differentialgleichung :

$$
\hat{x} \frac{d^{2} \hat{y}}{d \hat{x}^{2}}-(\hat{x}-a) \frac{d \hat{y}}{d \hat{x}}-b \hat{y}=0
$$

mit einer irregulären singulären Stelle $x=\infty$, wo die Zahl $a$ konstant ist. Für hinreichend grosse absolute Beträge von $x$ und $b$ ist es bezweckt die asymptotische Darstellung der Lösung des Systems (7.1) zu erledigen. Der Einfachheit halber behandeln wir den Fall, dass die Ungleichung

$$
M|\hat{x}|^{\frac{1}{2}}<|b|<\frac{1}{M^{\prime}}|\hat{x}|^{2}
$$

gilt, wo $M$ und $M^{\prime}$ hinreichend grosse positive Zahlen sind.

Wendet man die Transformation $\hat{x}=\lambda^{2} x, b=\mu$ auf (7.1) an, wo

$$
D: \quad|x-a|<r,(r<|a|)
$$

ist, wo die Grösse $a$ von Null verschieden ist, so geht (7.1) ins Folgende über :

$$
x \frac{d^{2} \hat{y}}{d \hat{x}^{2}}+\left(a-\lambda^{2} x\right) \frac{d \hat{y}}{d \hat{x}}-\lambda^{2} \mu^{2} \hat{y}=0,
$$

welche weiter durch die Transformation $\hat{y}=\hat{y}_{2}, d \hat{y} / d x=\lambda \mu \hat{y}_{1}$ ins Folgende über :

$$
d \hat{Y} / d x=\lambda \mu \hat{A}(x, \lambda, \mu) \hat{Y}
$$

worin

$$
\hat{A}(x, \lambda, \mu)=\left(\begin{array}{cc}
\frac{\lambda}{\mu}-\frac{a}{\lambda \mu x} & \frac{1}{x} \\
1 & 0
\end{array}\right)
$$

ist $Z$ wischen den Ordnungen $\lambda$ und $\mu$ muss die folgende Ungleichung gelten:

$$
U_{0}: \quad K|\lambda|<|\mu|<\frac{1}{K^{\prime}}|\lambda|^{4},
$$

woraus folgt : 


$$
n=1, n^{\prime}=1, n_{0}=1, n_{0}^{\prime}=4 \text {. }
$$

Um (7.3) zur Normalform reduzieren zu lassen, wenden wir die Transformation

$$
\hat{Y}=P_{1}\left(x, \lambda \mu^{-1}\right) Y
$$

mit

$$
\begin{gathered}
P_{1}(x, \nu)=\left(\begin{array}{cc}
\rho_{1}(x, \nu) & \rho_{2}(x, \nu) \\
1 & 1
\end{array}\right), \\
\rho_{1}(x, \nu), \rho_{2}(x, \nu)=\frac{\nu x \pm \sqrt{\nu^{2} x^{2}+4 x}}{2 x}
\end{gathered}
$$

auf (7.3) an, so geht (7.3) ins Folgende über :

$$
d Y / d x=\lambda \mu A(x, \lambda, \mu) Y,
$$

worin, wie eine einfache Berechnung sofort zeigt,

$$
\begin{aligned}
& A(x, \lambda, \mu)=\underset{0}{A}\left(x, \lambda \mu^{-1}\right)+K_{2} A\left(x, \lambda \mu^{-1}\right), \\
& \underset{0}{A}(x, \nu)=\left(\begin{array}{cc}
\rho_{1}(x, \nu) & 0 \\
0 & \rho_{2}(x, \nu)
\end{array}\right), \underset{2}{A}(x, \nu)=\left(\begin{array}{cc}
a_{11}(x, \nu) & \underset{2}{2} a_{12}(x, \nu) \\
a_{2}(x, \nu) & \underset{2}{a_{22}}(x, \nu)
\end{array}\right), \\
& \underset{2}{a_{11}}(x, \nu)=-\frac{\nu\left(a \rho_{1}+\rho_{1}^{\prime}\right)}{x\left(\rho_{1}-\rho_{2}\right)}, a_{2}(x, \nu)=-\frac{\nu\left(a \rho_{2}+\rho_{2}^{\prime}\right)}{x\left(\rho_{1}-\rho_{2}\right)}, \\
& a_{21}(x, \nu)=-\underset{2}{a_{11}}(x, \nu), \underset{2}{a_{22}}(x, \nu)=-a_{2}(x, \nu) \text {. }
\end{aligned}
$$

its ; daher ist das System (7.5) eine Normalform.

8. Die asymptotische Lösung. Wir betrachten nochmals das System (7.5) mit (7.6). Da das System (7.5) Normalform ist, so kann man durch ein ähnliches Verfahren wie bei $\S 2$ die asymptotische Lösung erledigen. In diesem Artikel behandeln wir den Fall $\sigma_{1}=3 / 2$. Nach Betrachtung von $m_{0}=2$ und $N_{0}=3$ gilt offenbar die Beziehung:

$$
\frac{\frac{n_{0}^{\prime}}{N_{0}}\left(N+m_{0}+1\right)-m}{\frac{n_{0}}{N_{0}}\left(N+m_{0}+1\right)+m^{\prime}}=\frac{3}{2},
$$

wenn wir $N=0$ annehmen. Führt man die Transformation

$$
Y=P(x, \lambda, \mu) Z
$$

mit 


$$
P(x, \lambda, \mu)=E+\sum_{r=1}^{2} K_{r} P\left(x, \lambda \mu^{-1}\right)
$$

in (7.5) ein, wo man nach (4.3), d. h. nach

$$
\begin{aligned}
& {[\underset{0}{A}(x, \nu) \underset{1}{P}(x, \nu)+\underset{1}{Q}(x, \nu) \underset{0}{A}(x, \nu)]_{j k}=0,(j \neq k ; j, k=1,2),} \\
& \underset{1}{P}(x, \nu)]_{j j}=0,(j=1,2), \\
& \underset{0}{A}(x, \nu) \underset{2}{P}(x, \nu)-\underset{2}{P}(x, \nu) \underset{0}{A}(x, \nu)+\underset{2}{A}(x, \nu)]_{j k}=0, \\
& \qquad(j \neq k ; j, k=1,2), \\
& \underset{2}{P}(x, \nu)]_{j j}=0,(j=1,2)
\end{aligned}
$$

$\underset{1}{P}(x, \nu)$ und $\underset{2}{P}(x, \nu)$ bestimmt, d. h.

$$
\begin{aligned}
& p_{1 k}(x, \nu)=0,(j, k=1,2), \\
& \underset{2}{p_{12}(x, \nu)}=-\frac{a_{12}(x, \nu)}{\rho_{1}(x, \nu)-\rho_{2}(x, \nu)}, \underset{2}{p_{21}}(x, \nu)=\frac{a_{21}(x, \nu)}{\rho_{1}(x, \nu)-\rho_{2}(x, \nu)} \\
& p_{2} j(x, \nu)=0,(j=1,2)
\end{aligned}
$$

ist, so geht (7.5) in

$$
d z_{j} / d x=\lambda \mu\left[f_{j}(x, \lambda, \mu) z_{j}+\sum_{k=1}^{2} b_{j k}(x, \lambda, \mu) z_{k}\right],(j=1,2)
$$

über, wo

$$
\begin{aligned}
& \underset{\mathbf{0}}{B}(x, \nu)=\left(\begin{array}{cc}
\rho_{1}(x, \nu) & 0 \\
0 & \rho_{2}(x, \nu)
\end{array}\right), \underset{1}{B}(x, \nu)=0, \\
& \underset{2}{B}(x, \nu)=\left(\begin{array}{cc}
a_{11}(x, \nu) & 0 \\
0 & \underset{2}{a_{22}}(x, \nu)
\end{array}\right), \underset{3}{B}(x, \nu)=0
\end{aligned}
$$

ist, welche durch die passende Transformation

$$
Z=\bar{P}(x, \lambda, \mu) \bar{Z}
$$

mit

$$
\bar{P}(x, \lambda, \mu) \approx E+\sum_{r=3}^{\infty} K_{r} \bar{P}\left(x, \lambda \mu^{-1}\right)
$$

uns zur Differentialgleichung 


$$
d \bar{Z} / d x=\lambda \mu[F(x, \lambda, \mu)+\bar{B}(x, \lambda, \mu)] \bar{Z}
$$

führt, welche weiter durch die passende Transformation

$$
\bar{Z}=\overline{\bar{P}}(x, \lambda, \mu) \overline{\bar{Z}}
$$

mit

$$
\overline{\bar{P}}(x, \lambda, \mu) \approx E+\sum_{r=1}^{\infty} \overline{\bar{P}}(x, \lambda, \mu)
$$

wo man nach (4.9) z. B. $\underset{\bar{P}}{1}(x, \lambda, \mu)=0$ annehmen kann, zu

$$
d \overline{\bar{Z}} / d x=\lambda \mu F(x, \lambda, \mu) \overline{\bar{Z}}
$$

wird, wo

$$
f_{1}(x, \lambda, \mu)=\rho_{1}\left(x, \lambda \mu^{-1}\right)+K_{2} a_{11}\left(x, \lambda \mu^{-1}\right), f_{2}(x, \lambda, \mu)=\rho_{2}\left(x, \lambda \mu^{-1}\right)+K_{2} a_{22}\left(x, \lambda \mu^{-1}\right)
$$

ist ; daher besitzt (7.3) eine für $\left(D, \Delta_{\lambda}, \Delta_{\mu}, U_{1}\right)$ reguläre eindeutige Lösung, die sich für $\lambda \rightarrow \infty, \mu \rightarrow \infty$ folgendermassen asymptotisch entwickeln lässt:

$$
\begin{aligned}
\hat{y}(x, \lambda, \mu)=\hat{y}_{2}(x, \lambda, \mu) \simeq C_{1} \exp \left[F_{1}(x, \lambda, \mu)\right] \\
\quad \times\left[1+K_{21}\left(p_{21}\left(x, \lambda \mu^{-1}\right)\left(\lambda \mu^{-1}\right)^{4}+\underset{2}{\bar{p}_{11}}\left(x, \lambda \mu^{-1}\right)\right)+\ldots . .\right],
\end{aligned}
$$

wo

$$
F_{1}(x, \lambda, \mu)=\lambda \mu \int\left(\rho_{1}\left(x, \lambda \mu^{-1}\right)+\underset{2}{\left.K_{2} a_{11}\left(x, \lambda \mu^{-1}\right)\right) d x}\right.
$$

ist. Da man, wie eine kurze Berechung zeigt,

$$
\begin{aligned}
& F_{1}(x, \lambda, \mu)=\lambda \mu\left[x \rho_{1}\left(x, \lambda \mu^{-1}\right)-\frac{\mu}{\lambda} \log \left(-\frac{\rho_{2}\left(x, \lambda \mu^{-1}\right)}{\rho_{1}\left(x, \lambda \mu^{-1}\right)}\right)\right. \\
& \left.\quad+K_{2}\left(-\frac{a \lambda}{2 \mu} \log \frac{x \rho_{1}\left(x, \lambda \mu^{-1}\right)}{\rho_{2}\left(x, \lambda \mu^{-1}\right)}+\frac{\lambda^{3}}{8 \mu^{3}} \log \left(2 \rho_{1}\left(x, \lambda \mu^{-1}\right)-\lambda \mu^{-1}\right)\right)-\frac{\lambda}{4 x \mu}\right]
\end{aligned}
$$

erhält, so besitzt (7.3) eine eindeutige Lösung $\hat{y}(x, \lambda, \mu)$, welche für $\left(D, \Delta_{\lambda}, \Delta_{\mu}\right.$, $\left.U_{1}\right)$ regulär ist, und sich folgendermassen asymptotisch entwickeln lässt:

$$
\begin{array}{r}
\hat{y}(x, \lambda, \mu) \simeq C_{1} \exp \left[\lambda \mu x \rho_{1}\left(x, \lambda \mu^{-1}\right)-\frac{1}{4 x}\right]\left(-\frac{\rho_{1}\left(x, \lambda \mu^{-1}\right)}{\rho_{2}\left(x, \lambda \mu^{-1}\right)}\right)^{\mu^{2}}\left(\frac{\rho_{2}\left(x, \lambda \mu^{-1}\right)}{x \rho_{1}\left(x, \lambda \mu^{-1}\right)}\right)^{\frac{a}{2}} \\
\times\left(2 \rho_{1}\left(x, \lambda \mu^{-1}\right)-\lambda \mu^{-1}\right)\left[1+\sum_{r=2}^{\infty} K_{r, 1} p_{r}\left(x, \lambda \mu^{-1}\right)\right],
\end{array}
$$

wo 


$$
U_{1}: \quad K|\lambda|<|\mu|<\frac{1}{K^{\prime}}|\lambda|^{\frac{3}{2}}{ }^{16)}
$$

ist. $^{17)}$

\section{LITERATURVERZEICHNIS}

[1] M. HuKuHARA, Sur les propriétés asymptotiques des solutions d'un système d'équations différentielles linéaires contenant un paramétre, Memo. Fac. Eng. Kyûshû Imp. Univ., 8(1937), 249-280.

[2] M. IWANO, Ein Differentialgleichungssystem mit zwei Parametern, (auf französisch), (1960), (Unter der Beitrag).

[3] T. INUI, Kugelfunktionen, Zylinderfunktionen und Hypergeometrische Funktionen, (1948), (Kawade-Syobo, Tokyo).

[4] K. TAKAhASH, Ein System von linearen homogenen Differentialgleichungen, welche auf zwei Parametern abhängen, Tôhoku Math. J., 8(1956), 258-267.

[5] K. TAKAHASH, Über eine erweiterte asymptotische Darstellung der Lösung eines Systems von homogenen linearen Differentialgleichungen, welche von zwei Parametern abhängen, J. Fac. Sci. Univ. Tokyo, 8(1959), 1-73.

UNIVERSITÄT DER LANDWIRTSCHAFT UND TECHNOLOGIE ZU TOKIO.

16) Für $U_{2}$ von $\S 2$ an Stelle von $U_{1}$ besitzt (7.3) eine asymptotische Löung.

17) Wir denken uns die Whittakersche Differentialgleichung:

(W) $\frac{d^{2} W}{d t^{2}}+\left[-\frac{1}{4}+\frac{K}{t}-\left(m^{2}-\frac{1}{4}\right) t^{2}\right] W=0$

mit einer irregulären singulären Stelle $t=\infty$, wo $m$ eine Konstante, und $K$ ein Parameter ist. Wendet man die Transformation $t=\lambda^{2} x$ auf $(W)$ an, so geht $(W)$ ins Folgende über :

(W') $\frac{d^{2} W}{d x^{2}}+\left[-\frac{1}{4} \lambda^{4}+\frac{a-2 \mu^{2}}{2 x} \lambda^{2}-\frac{a(a-2)}{4 x^{2}}\right] W=0$.

Der Einfachheit halber behandeln wir nur den Fall, dass die Ungleichung

$$
U_{0}: \quad K|\lambda|<|\mu|<\frac{1}{K^{\prime}}|\lambda|^{4}
$$

gilt. Wendet man die Transformation

$$
W=\exp \left[-\frac{\lambda^{2} x}{2}\right]\left(\lambda^{2} x\right)^{\frac{a}{2}} y
$$

auf $\left(W^{\prime}\right)$ an, so geht $\left(W^{\prime}\right)$ ins Folgende über :

(W'I) $\quad x \frac{d^{2} y}{d x^{2}}+\left(a-\lambda^{2} x\right) \frac{d y}{d x}-\lambda^{2} \mu^{2} y=0$,

welche die konfluente hypergeometrische Differentialgleichung ist; daher kann man nach $\$ 4$ die asymptotische Lösung von ( $\left.W^{\prime \prime}\right)$ erfinden. 